\title{
RECHERCHE ET DOSAGE DU DIACÉTYLE DANS LES BEURRES ( 1 )
}

\author{
par \\ MM. R. DEHOVE et L. DESSIRIER \\ Ingénieurs Chimistes I. C. L., \\ Chimistes au Laboratoire Municipal de Lille.
}

L'article 9 de la loi du 2 juillet 1935 interdit l'addition de parfums artificiels dans les beurres.

Le plus employé est le diacétyle, d'abord à cause de sa bonne odeur de beurre frais en solution très étendue, ensuite parce qu'on peut le rencontrer dans les beurres en petites quantités.

Les prélèvements de beurre mis à la disposition du Laboratoire pour la recherche des fraudes et falsifications étant de 150 grammes environ, il importe de trouver une méthode permettant de n'opérer que sur une faible quantité de beurre, n'excédant pas 50 grammes.

De plus, comme la dose de diacétyle qui peut exister naturellement dans les beurres n'est que de quelques milligrammes par kilo, il devient nécessaire d'évaluer la quantité de ce produit avec une précision au moins égale au milligramme par kilo.

Il nous semble, dès à présent, possible d'affirmer, en accord avec plusieurs auteurs qui ont étudié aussi la question, qu'un beurre contenant plus de 5 milligrammes de diacétyle par kilo peut être considéré comme ayant été additionné de ce produit.

En dessous de cette quantité, il peut y avoir doute.

Toutefois, nous poursuivons une étude statistique des beurres de la région du Nord pour déterminer la dose maxima de diacétyle qu'ils peuvent contenir naturellement.

A notre connaissance, aucune des méthodes préconisées jusqu'à ce jour ne permet une précision de l'ordre du milligramme par kilo en n'opérant que sur 50 grammes de beurre.

Le dosage quantitatif du diacétyle comprend deux phases :

10 L'extraction;

$2^{\circ}$ La caractérisation et le dosage du diacétyle extrait.

$1^{\circ}$ Extraction. - L'extraction à l'alcool éthylique, telle que l'ont décrite MM. GUILLot et VIzern, ne permet pas d'extraire la totalité du diacétyle de la prise d'essai.

En effet, nous avons caractérisé encore très nettement le diacétyle dans un beurre diacétylé à dessein, même après application de ce mode d'extraction (2).

(1) Annales des falsifications et des fraudes, No 342 , juin 1937.

(2) L'extraction à la vapeur d'eau, telle que nous la pratiquons, permet, au contraire, d'extraire la totalité du diacétyle. 
$2^{\circ}$ Caractérisation du diacétyle. - On a proposé plusieurs réactions permettant de caractériser le diacétyle, et, éventuellement, de le doser quantitativement.

Pour avoir une précisión de un milligramme par kilo en partant de 50 grammes de beurre, il faut que nous appliquions une réaction permettant de déceler $0 \mathrm{mmgr}$. 05 dans la prise d'essai.

a) Réaction de LEMOIGNe, modifiée par GuILLOT et VIzERN. On transforme le diacétyle en diméthylglyoxime que l'on caractérise par son sel de nickel. En suivant le procédé décrit par Guillot et Vizern nous ne pouvons déceler que quelques milligrammes par kilo, la prise d'essai étant de 50 grammes, ce qui n'est pas assez précis pour le cas qui nous concerne.

b) Formation de la quinoxaline. - Cette réaction est préconisée par M. Pren qui part de la mèta-para-toluilène diamine (Voir Le Lait, $\mathrm{n}^{\circ} 152$, février 1936, page 136). Il en a déduit une méthode colorimétrique de dosage ; malheureusement cette méthode nécessite une quantité de beurre trop grande, vu sa sensibilité. Elle ne permet, en effet, que de déceler 4 milligrammes par kilo en partant de 50 grammes de beurre.

c) Formation de la xyloquinone. La sensibilité étant de $1 / 20.000^{\mathrm{e}}$, cette réaction ne permet une précision que de 20 milligrammes par kilo dans les conditions où nous opérons.

De cette courte étude, il s'ensuit que nous ne pouvons appliquer ni l'extraction à l'alcool, ni aucune des réactions proposées jusqu'alors.

Mais on peut essayer néanmoins :

A. - Soit de rendre les réactions précédentes plus sensibles;

B. - Soit d'en mettre au point d'assez sensibles pour les appliquer dans notre cas.

A. - Voici la méthode que nous proposons pour doser quantitativement le diacétyle dans les beurres.

10 Principe. - 50 grammes de

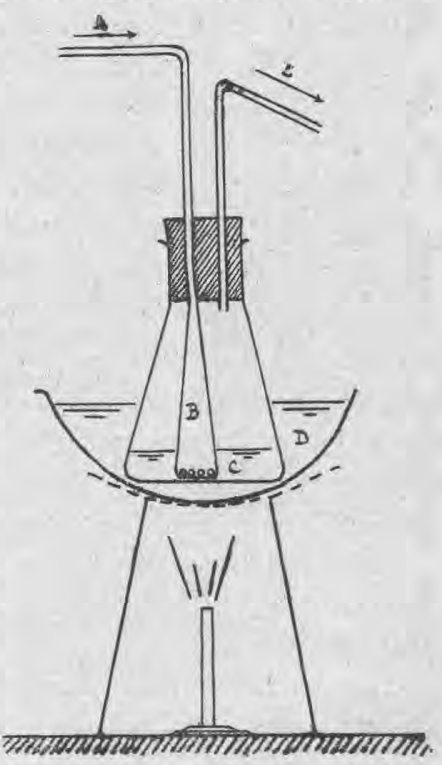

Sohéma de l'appareil à extraction (Barbotteur).

A : Courant de vapeur d'eau.

B : Tube injecteur.

C : Beurre à essayer.

D : Bain de chlorure de calcium.

$\mathrm{E}$ : Vers le réfrigérant.

beurre sont traités par la vapeur d'eau, on recueille 20 centimètres cubes de distillat et on transforme le diacétyle obtenu en diméthylglyoxime que l'on précipite par un sel de nickel. On évapore à sec 
et on sépare le diméthylglyoximate de nickel des impuretés et des excès de réactif au moyen de chloroforme qui ne dissout que le diméthylglyoximate de nickel. On évalue ensuite la quantité de diacétyle par comparaison.

$2^{\circ}$ Appareillage. - Il comprend :

a) Un ballon producteur de vapeur d'eau. - Ce ballon est muni d'un bouchon percé de deux trous, l'un pour le passage de la vapeur, l'autre portant un tube à robinet que l'on peut ouvrir ou fermer à volonté et qui sert à la fin de chaque opération à empêcher les aspirations de liquides d'un récipient dans l'autre.

b) Un tube injecteur de vapeur, plongeant jusqu'au fond de la fiole d'Erlenmeyer et dont l'extrémité plate est percée sur le pourtour de petits trous permettant la répartition régulière de la vapeur dans le beurre à examiner (voir figure).

c) Une fiole d'Erlenmeyer de $250 \mathrm{~cm}^{3}$ à large goulot portant un bouchon à deux trous, dont l'un sert à l'arrivée de la vapeur et l'autre au départ de la vapeur chargée de diacétyle. Cette fiole plonge dans une capsule contenant une solution de chlorure de calcium pour la maintenir à une température légèrement supérieure à $100^{\circ}$.

d) Un réfrigérant à eau muni à son extrémité d'un tube effilé plongeant dans une petite quantité d'eau defaçon à éviter des pertes en diacétyle qui aurait pu passer à l'état gazeux à travers le réfrigérant tout au début de l'opération.

$3^{\circ}$ Mode opératoire. - 50 grammes de beurre à examiner sont placés dans la fiole d'Erlenmeyer. On maintient le bain de chlorure de calcium à une température légèrement supérieure à $100^{\circ}$ pour éviter la condensation du courant de vapeur émis par le ballon producteur. On distille ainsi $15 \mathrm{~cm}^{3}$ et comme on a pris la précaution de faire barboter les premières vapeurs dans $5 \mathrm{~cm}^{3} \mathrm{~d}^{\prime}$ eau, on a finalement un volume de $20 \mathrm{~cm}^{3}$ renfermant tout le diacétyle de la prise d'essai.

On ouvre alors le robinet du ballon producteur de vapeur pour éviter les aspirations de liquide et, sur le distillat, on dose le diacétyle.

$4^{\circ}$ Caractérisation et dosage du diacétyle. - Les $20 \mathrm{~cm}^{3}$ de distillat sont traités par $1 \mathrm{~cm}^{3}$ de chlorhydrate d'hydroxylamine à $10 \%$, puis par $2 \mathrm{~cm}^{3}$ de soude normale. On agite une minute et on ajoute $1 \mathrm{~cm}^{3}$ d'une solution de sulfate de nickel à $0 \mathrm{~g} .2 \%$, puis $0 \mathrm{~cm}^{3} 6$ d'acide acétique normal.

On transvase le tout dans une capsule de porcelaine et on évapore à sec au bain-marie.

Le résidu est repris 3 fois par $2 \mathrm{~cm}^{3}$ environ de chloroforme en ayant soin d'agiter la masse avec un agitateur pointu. On filtre sur 
un filtre sans pli, on lave le filtre avec une petite quantité de chloroforme et on réunit filtrats et liquide de lavage dans une petite capsule de porcelaine blanche à fond plat. On évapore le chloroforme au bain-marie et le diméthylglyoximate de nickel se dépose en rouge violacé plus ou moins intense suivant la quantité de diacétyle contenue initialement dans le beurre.

On fait une série de témoins en partant de diacétyle pur de façon à avoir une gamme de colorations correspondant à des quantités de diacétyle variant de $0 \mathrm{mgr}$. 05 à $0 \mathrm{mgr}$. 5, telles que : 0 mgr. $05-0$ mgr. $10-0 \mathrm{mgr}$. $15-0 \mathrm{mgr}$. $45-0 \mathrm{mgr}$. 50, ce qui donne des quantités de diacétyle variant de 1 à 10 milligrammes par kilo.

Par comparaison avec cette échelle de témoins, il est très aisé d'évaluer les quantités de diacétyle à $0 \mathrm{mgr}$. 025 près, c'est-à-dire avec une précision de 0 mgr. 5 par kilo, puisque la quantité de beurre mise en jeu n'est que de 50 grammes.

B. - Les réactions colorées du diacétyle sont en très grand nombre et nous en avons cherché de plus sensibles que celles citées plus haut (quinoxaline et xyloquinone) permettant un dosage colorimétrique à la fois plus scientifique et plus sensible que la méthode précédente et ne nécessitant cependant qu'une minime quantité de beurre.

Nous avons observé que la pyrocatéchine et la résorcine donnaient en milieu sulfurique une coloration très intense avec des traces de diacétyle, et nous étudions actuellement un procédé de dosage basé sur l'une de ces réactions.

Nota. - La presque totalité des beurres renferme naturellement une petite quantité d'acétyl-méthyl-carbinol qu'il peut être intéressant de doser. Par oxydation ménagée $\left(10 \mathrm{~cm}^{3}\right.$ de perchlorure de fer à $25 \%$ ) on le transforme faeilement en diacétyle que l'on dose par la méthode citée précédemment.

\section{BIBLIOGRAPHIE ANALYTIQUE}

\section{LES LIVRES}

13de Beretning fra statens Forsogsmejeri (13e Rapport de la Laiterie d'essais de l'Etat, Copenhague, 1937). Jknsen (J.) et Herning (S.). - Désinfection chez les producteurs de lait par des préparations à base de chlore. 1 br. de 45 pages.

Prix : 1 cour. danoise.

Les recherches dont les résultats sont résumés ci-dessous ont été effectuées pendant l'été 1936. Auparavant, le Professeur Sôneke KNudsen ainsi 